\title{
Sachregister zu Band 66
}

Die fett gedruckten Zahlen bezeichnen Eigenarbeiten. Bb. = Buchbesprechung.

A

Ablatio retinae 101, 102398

Anatomie der freien - 285

bei großen Aderhautkolobomen 462

totale - bei Chorioiditis exsuda-tiva serosa sympathica 169

- $\quad$ operative Heilung der - 488Achsenmyopie 381Aderhaut s. Chorioidea.Adrenalin,

Wirkung des - auf das

glaukomatöse Auge 381 Akkommodation, kardiale - und respiratorischer Reflex während der 386 Akkommodationshilf en 188 Albinismus 459, 474 Amblyopie, rezidivierende 398 Angiom, kavernöses - der Chorioidea 359 Aniridie 457

- $\quad$ vollständige - nach Trauma 369Anisokorie, Bedeutung der - bei

homogener Hemianopsie 300 Anophthalmus 451 Aphakie mit guter Sehschärfe 399 Arcus

senilis corneae lipiodes

337- 338 Arteria hyaloideapersistens 460 Asymbolie, optische 261

- $\quad$ die pathologischen Grundlagender optischen - 287

Atmung, Einfluß der psychoopti-schen Sphäre und der psycho-motorischen Zentren auf die -

373

Atropininjektion, retrobulbäre - bei Sehnervenatrophie 390

Augapfel s. Bulbus

Auge, Entwicklung des - 206 (Bb.)

Augendiagnose, Nachprüfung der -484

Augendrehpunkt, Bestimmung des -186

Augendruck s. Druck

Augenentzündung und Augendruck 487

- Chemotherapie der traumatischenintraokulären - 392

Augenflüssigkeiten, Übertrittim-muno-spezifischer Stoffe in die - 170

Augenhintergrund, stereoskopi-sche Photographien des - 194

Augenmneres, Erkrankungen des - im Zusammenhang mit Erkrankungen des Ohrs 293

Augenlider s. Lider

Augenmuskelkrampf, konjugier-ter - bei Enzephalitis epidemica 412

Augenmuskeln 84 (Bericht)

Anomalien der äußeren - 455

metastatische Myositis der - 191 Augenspiegel, reflexloser stereo-

skopischer Hand 202

Augentropfen, Keimgehalt von -

100 Augenverband, durchsichtiger -

aus Zellon 201 Augenverletzungen 112, 379

- $\quad$ Begutachtung der - mit Rück-sicht auf die Arbeiterversicherung 
$39 \theta$ Automobilführer, Anforderungen

für die Sehschärfe der - in der

Schweiz no Autonome Innervation d. Auges,

Beziehung der Blut-Kammer-

wasserschranke zur - 141 Axenfeld-Krukenbergsche

Pigmentspindel 456

B

Barlowsche Krankheit, Augen-

veränderungen bei der - 379 Behrsches Phänomen 300 Beleuchtungsst $3 / 4$, rke, Abhängig-

keit der Sehschärfe von der - 288 Bindehaut s. Conjunctiva Blepharochalasisähnliche Lid-

schwellung 475 Blindenstatistik von Köln 228 Blindenwesen 418 Blindheit, sofort einsetzende bei

Quetschung des Thorax 205 Blutdruck, Wechselbeziehungen

zwischen Augenerkrankungen und

$-183,400$

- $\quad$ Beziehungen des - zum Augendruck und zur Hornhautpulsation144

Blut-Kammerwasserschranke in Beziehung zur autonomen Innervation des Auges, insbesondere zum Gefäßsympathicus 141

B1 u t u n g, expulsive 404

- $\quad$ in atrophischen Augen 358, 359

494

Sachregister zu Bd. 66.

Böser Blick, Mittel gegen den -

420 (Bb.) Brechzustände des Auges, ent-

wicklungsgeschichtliche Auffas-

sung der - 128 Bulbusalbinismus, genetischer

Zusammenhang zwischen heredi-

tärem Nystagmus und - 98 Bulbusverletzung, perforierende

487 Buphthalmus und endokrine Stö-

rungen 380

C Calmettescb.es Tuberkulose-

virus BCG 120 Compimeter 287 Canaliculus lacrimalis, Epitheli-

oma papillare des - 399 Chalazion 329 Chemische Industrie, Augenschä-

digungen in der - 283 Chemosis als erstes Zeichen eines

Sarkoma chorioideae 265 Chemotherapie der traumatischen

intraokulären Entzündungen 392

Cholesteringehalt des Blutes beim

Arcus lipoides corneae senilis 338

Chorioidea, pathologische Anato-

mie der $-355 \mathrm{ff}$.

-Blutungen der - nach Starope-rationen 281

Melanosarkome der - 157, 162

Sarkom der - 252, 262

Chemosis als erstes Zeichen eines Sarkoms des - 265

Chorioiditis, experimentelle sym-pathische 168 
- $\quad$ exsudativa serosa sympathica mittotaler Netzhautablösung 169

- $\quad$ tuberculosa mit Sternfigur 482Chromatische Erregung, Tonus-

valenz der - bei der optomoto-

rischen Reaktion 135 Conjunctiva, pathologische Ana-

tomie der-33iff.a-Kapselbakterien im sack 486

Scharlachrotfärbung der - in

Melanose der - 195

Erkrankung der - bei Erythema nodosum 323

Bildung von Pseudomembranen an den - und eines Granuloms am Lide 204

Conjunctivitis phlyctaenula-

ris, experimentelle 121 Cornea, pathologische Anatomie der

- 336ff. - Darstellung der kranken - im

Reflexbild 159

- $\quad$ Blutdruck, Augendruck und Pulsation der - 144

- $\quad$ periphere Ektasie der - 166

Cornea, hereditäre rezidivierende Erosion der - 309

Follikelbildung auf der - bei Frühjahrskatarrh 197

Scharlachrotfärbungdes Ill

-mikroskop 384

-naht bei der Starextraktion 396

-trübungen, angeborene 455

- angeborene nach Zangen-geburt 484

- $\quad$ - knötchenförmige 342Corpus ciliare, Melanokarzinom

des -482 Crista lacrimalis anterior ac-

cessoria 335 Cysticercus cellulosae subreti*

nalis 252 Czermak-v. Hippelsche Netz-

hauterkrankung 1

v

Dakryokanalikulitis cystica

336

Dämmerungszittern 92 Deckf alt en bildung bei der Ptosis-

operation 165 Dermoidzysten 454 Descemetsche Membran, Früh-

operation der - 343 Diathermie, Elektrodenimprovisa-

tion für - 108 Diplopie monokuläre 266 Doppelf okusglas 188 Druck, intraokularer und Augenentzündung 487

Einfluß der Schwangerschaft auf den - 382, 386

tiefe randständige Exkavation ohne Steigerung des - 403

Beziehungen des - zum Blutdruck und zur Hornhautpulsation 144

Dunkeladaptation, Verlauf der - nach vorheriger Helladaptation an verschiedene spektrale

Lichter 137, 425

EEisenkies, Augenverletzungen

durch - 379 Eklampsie, spastische und tetani-

sche Netzhautgefäßveränderun-

gen bei - 178 Ektopia lentis 457 Elektrodenimprovisation für

Augendiathermie 108 Elektrolytgehalt von Serum, Se-

rumultrafiltrat und Kammerwas- 
ser 138 Endokrine Befunde bei Retinitis

pigmentosa 487 Endokrine Störungen und Buphthalmus 380

Sachregister zu Bd. 66.

495

Entropium senile, Tarsektomie

mit implantation von Ohrknorpel

gegen - 396 Entwicklungsanomalien, ange-

borene - 450 (Bericht) Enzephalitis epidemica, kon-

jugierter Augenmuskelkrampf bei

$-\quad 412$

- Okulomotoriuslähmung bei-382Enzephalozele 192Ephetonin 432

Epitarsus 454

Epithelf as er system der Hornhaut

339

Epithelioma papillare des Cana-

liculus lacrymalis 399 Erbsche Krankheit mit Herab-

setzung des Sehvermögens 385 Erythema nodosum, Erkran-

kung der Bmdehaut bei - 323 Exkavation, glaukomatöse,

Knochenbildung innerhalb einer

$-\quad 239$

- $\quad$ tiefe randständige - mit Seh-nervenatrophie ohne Drucksteige-rung 403

Exophthalmus, pulsierender 252, 259

$\mathrm{F}$

Farbensinnstörungen, angebo-

rene 291 Feuerar beiter, gewerbliche Lin-

senschädigungen bei - 164 Flocculus iridis, abgebröckelter-

in der Vorderkammer 459 Flimmerskotom 421 Flüssigkeitsbewegungen im

Auge 137, 148, 149 Fremdkörper in der Linse 410 Frühj ahrskatarrh 330

- $\quad$ Follikelbildung auf der Hornhautbei - 197

G

Gef äßsympathikus, Beziehung

der Blut-Kammerwasserschranke

zum - 141 Gegenrollung der Augen 93 Gehirntumor, einseitige Papillitis

bei - 263 Gehörstörungen und Augener-

krankungen 293, 481 Geschwülste des Auges 360 Gesichtsatrophie, strichförmige

- $\quad$ und Auge 115Gesichtsnervenlähmung, irreparable - durch gewerbliche Ver-giftung 491

Gewebezüchtung und Keratitis

158, 164 Glaskörper, pathologische Anato-

mie des - 347 if.

Glaskörperersatz 182 Glaskörperquellung 142 Glaukom 143, 149, 290, $379 \mathrm{ft}$.

Linsenkapselhäutchen-103, 105

Veränderungen der Ziliargefäße bei - 196

und Irisatrophie 457

und Naevus flammeus 264, 267

Wirkung der Miotika auf das - 
H5 Glaukomoperation nach La-grange 475

- wiederholte 393

Gliom, Differentialdiagnose zwi-

schen Pseudogliom und - 381 Granulom am Lide, rezidivieren-

des bei pseudomembranbildender

Conjunctivitis 204 Granulose, Röntgentherapie der-

441

H.

Haárfärbemittel, Augenerkran-kungen durch - 385

Hämangioendotheliom der Conjunctiva 334

Hämangiom der Orbita, durch Krönleinsche Operation entfernt 481

- $\quad$ des Ziliarkörpers 359Handaugenspiegel, reflexloser

stereoskopischer 202 Hebelnystagmographie 97 Hemianopsie, Bedeutung der Ani-

sokorie bei homonymer - 300 Herpes 166, 167

der Hornhaut 341, 342

u. sympathische Ophthalmie 364 Histiozyten, Rolle der - bei der

degenerativen Verfettung des Bul-bus 158, 163

Höhlenbildung im Sehnerven 127

Hornerscher Symptomenkom-plex 378

Hornhaut s. Cornea

Hyaline Entartung der Conjunctiva 331

Hydrafthrose bei hereditärer Syphilis 385

Hydrophthalmus congenitus 192, 452

Hypercholesterinämie, Bezie-hungen der - zum Glaukom 383

Hypertonie und Auge 183, 400

- $\quad$ Beziehungen der - zu Gefäßver-änderungen und Blutungen der -194

Hypopyon-Iritis 243

- $\quad$-Uveitis 352Hypotonie, hochgradige - des

Augapfels bei Verbindung des Schlemmschen Kanals und der Vorderkammer nach außen 487

496

Sachregister zu Bd. 66.

I

Ichthyosis 408

- corneae 267Immunospezifische Stoffe,

Übertritt der - in die Augen-

flüssigkeiten 170 Implantationszyste, sklerale epi-

theliale 347 Innere Sekretion und Augenleiden

386 Insulin, Einfluß des - auf das

Auge 380 Iridozyklitis, Zirkulationslücke

bei - 107 Iris, pathologische Anatomie der -

$350 \mathrm{ff}$.

angiomatöse Veränderungen der - mit Glaukom 290

Polykorie bei essentieller Atrophie der - 267

Tuberkulose der - 409 Irisatrophie und Glaukom 457 Iritis, Hypopyon-I. 243 
Ursache und Schicksal der - 181

- $\quad$ haemorrhagica, Röntgenbestrah-lung bei - 397

$\mathrm{K}$

Kammerwasser, Elektrolytgehalt

des - 138 Kammerwasserbildung 140, 147 Kapselbakterien im Bindehaut-

sack 486 Kardialer Reflex während der

Konvergenz und Akkommodation

386 Karzinome der Bindehaut 333, 334

- $\quad$-metastasen in beiden Augen, vomMagen ausgehend 447

Katarakt, angeborene 459

- $\quad$ calcarea 408

experimenteller Röntgenstrahlen-K. 289

Sonnenblumenstar 106

- pathologische Anatomie der-366Katarakt-Embryonalnähte der

Linse 409 Kataraktoperation 398, 409

- $\quad$ lineare Extraktion 395

- Hornhautnaht und runde Pupille bei der - 396

- Chorioidealblutungen nach - 281

- $\quad$ Folgen der Wundsprengungennach - 396

Kavernom der Orbita 252 Keimgehalt von Augentropfen 100 Keratitis, pathologische Anatomie der $-339,340,341$

im Anschluß an Herpes 167

und Gewebezüchtung 158, 164

Röntgenbehandlung der -419

parenchymatosa bei Schlafkrank-heit 175

Keratitis parenchymatosa, Be-handlung der - 176

- $\quad$ - Behandlung der - mit Impf-malaria 316

- phlyctaenularis, experimentellei 21

profunda 408

superficialis punctata 480 Keratokonus 409 Keratoplastik 345 Kinematographische Aufnahmen von Augenoperationen 194 Knochenbildung, intraokulare399

- $\quad$ in der Linse 367

- $\quad$ innerhalb einer glaukomatösenExkavation 239

Knochenbrüchigkeit und blaue

Sklera 397, 456 Kobaltlampe 180, 193 Kolobome, Ontogenese der - 460,

461

- $\quad$ der Augenlider 454

- der Makulagegend 414Konkrementbildung im oberen

Tränenröhrchen 485 Konvergenz, kardialer und respira-

torischer Reflex während der -

386 Kopflichtbäder, Sehstörungen

nach - 408 Körperbau und Refraktion 50 Korrigierende Gläser vor Augen-

prothesen 83 Krönleinsche Operation zur Ent-

fernung eines Haemangioma or-

bitae 481

L Lepra, mikroskopische Präparate 
eines Bulbus von - 267 Leukosarkom der Bindehaut 334 Lichen conjunctivae 332

Lichtbehandlung des Auges 275,

445

- $\quad$ Dosisbestimmung bei der - 174Lider, pathologische Anatomie der

- $\quad 328 \mathrm{ft}$.

- Aplasie des - 265

akutes anaphylaktisches Odem der -474

-kolobome 454

Linse, pathologische Anatomie der

- $\quad 347 \mathrm{ff}$.

Mißbildungen der - mit Mikr-ophthalmus 282

angeborene Ringstar- - 192

gewerbliche Schädigungen der - bei Feuerarbeitern 164

Linsenkapselhäutchenglaukom 103, 105

Linsenluxation unter der Bindehaut 409

Lumbalanästhesie, Augenmuskel-lähmungen infolge von - 383, 384

Lupus der Bindehaut 484

Sachregister

$\mathrm{M}$

Macula, Kolobom der M.-Gegend

4X4. 463

- $\quad$ Vererbung der Kolobome der -

253 Magische Heil- und Schutzmit-

tel $420(\mathrm{Bb}$,) Malariabehandlung der Keratitis

parenchymatosa 316

- der luetischen Sehnervenatrophie381

Megalokornea 399, 455

Melancholieinfolge der irrigen Vor-stellung des Schadens eines in der Augenhöhle fest

verheilten Splitters 416

Melanokarzinom des Ziliarkörpers 482

Melanosarkome, chorioideale 157, 162

Melanose der Bindehaut 195

Mikrophthalmus 451

- mit Linsenmißbildungen 282

Mikropsie 375

Milzbrandödem 253

Miotika, Wirkung der - auf das Glaukomauge 145

Mißbildungen 450 (Bericht)

Modelle, Einführung neuer - für Forschung und Unterricht 192

Muskelvorlagerung 209

Myopie 267, 269ft., 381

- $\quad$ Genese der - 128, 130Myositis, metastatische - der

äußeren Augenmuskulatur 191 N Nachbilder 188

Naevus und Glaukom 264, 267, 290 Naevuskarzinom 346 Nasennebenhöhlen und Aúge 268, 411 Nematode, subkonjunktivale 196 Nervozidin 145 Xervus oculomotorius, dissoziierte Lähmungen des -380 
- $\quad$ beiderseitige Lähmung des - beiEncephalitis epidemica 382

Nervus opticus, Erkrankungen des Stammes des - 410

Höhlenbildung im - 127

Kolobome des -461

- V Veränderungen des - nach Kopf-traumen 369

Beeinflussung der Topographie des Kopfes des - nach Elliot-scher Trepanation 256

-Atr op hie bei tiefer randständi-ger Exkavation ohne Drucksteige-rung 403

- Malariabehandlung bei lueti-scher - 381

- Behandlung der tabischen - 387. 390

Zeitschñft für Augenheilkunde. Bd. 66. Heft 6.

zu Bd. 66. 497

Netzhaut s. Retina. Neuritis optica, Trepanation des Sinus sphenoidalis bei - 292

- $\quad$ r etrobulbari s 411

- $\quad$ - Pathogenese und Klinik der

$-\quad 249$

- rhinogene 124

- traumatica 397 Nystagmus 94ft., 375

0

Oculopapilläre sympathische Fasern, Verlauf der - 262

$\mathrm{O}$ h r , Erkrankungen des Augeninnern im Zusammenhang mit Erkrankungen des - 293, 481

Ölzysten, prälakrymale 383

Operation en, kinemato, graphische Aufnahmen von - 194 - endogene und metastatische Ophthalmie nach -123

Ophthalmia sympathica 347Íf.

Ophthalmie, endogene und metastatische - nach Augenopera-tionen 123

Ophthalmologische Gesell-schaft in Wien, 25jah.ri.ges Be-stehen der - 468

Ophthalmomalazie, Genese der

- 386Ophthalmoplegia internuclea-

ris 91 Optisch-ophthalmologische

Genauigkeit 185 Optomotorische Reaktion, To-nusvalenz der chromatischen Er-regung bei der135 Orbitopalpebralzyste 407 Osteochondrom des Unterlides 330 Oxydations - Reduktions Potential der Netzhaut 177

$\mathrm{P}$

Pannus, Behandlung des trachoma-tösen - - mit clem Sekret der trachomatösen Bindehaut 399

Papilla nervi optici, Aplasie der

- 460

Papillitis, einseitige - bei Hirn-tumor 263

- $\quad$ tuberculosa mit Sternfigur 482Parinaudsche Conjunctivitis

323 Parkinsonsche Krankheit, Au-

gensymptome bei - 377 Pathologie und pathologische

Anatomie des Auges 292 (Bb.),

328 (Bericht) Pfefferminzölschädigung des

Auges 255 Pigmentsegel 350 Pigmentspindel, Axenfeld-Kru-

kenbergsche -456

33 
3Г zu Bd. 66.

498 Sachregist $€$

Phlyktäne 342

PI as mom 331

Plastik, Früh-P. mit Mundschleim-

haut bei Verätzungen 263 P o 1 y k o u.r i e bei essentieller Atrophia

iridis 267 Polyzythämie, Störungen der

Ñetzhautzirkulation bei - 196 Prothesen, korrigierende Gläser vor

$-\quad 83$

- $\quad$ zum Schutze bei Röntgen- undRadiumbestrahlung 487

Pseudogliom, Differential diagnose zwischen Gliom und - 381

- $\quad$ congenitum, familiäres Auftretendes - 368

Pseudoluxation der Linse 378

Pseud osklerose, Pigmenteinlage-rung in die Descemetsche Mem-bran bei - 367

Psikain-N. 261

Psychologische Momente, Ein-fluß der - auf die farbigen Schwellen der anomalen Trichromaten 133

Pterygium 335

Ptosisbrille 165

Ptosisoperation, Deckfaltenbil-dung bei der - 165

Piipillenstörungen, anatomische Grundlage der - 157

Pyozyaneusinfektion der Horn-haut 345

Pyrit, Augenverletzungen durch - 379

$\mathrm{R}$

Radiumbestrahlung, Prothese

zum Schutze gegen 487 Raumwahrnehmung und Tonus

190 Reflektographie, Apparatur zur 200

Reflexbild, Darstellung der kran-

ken Hornhaut im - 159 Refraktion und Körperbau 50 Respiratorischer Reflex während der Konvergenz und Akkom-

modation 386 Retina, funktionelle Gliederung und

Einteilung der - 85, 113

- $\quad$ eigenartiges Krankheitsbild der-

254

Czermak - v. Hippelsche Erkran-kung der - 1

- spastische und tetanische Gefäß-veränderungen der - bei Eklam-psie 178

- Beziehungen der Hypertonie zuGefäßveränderungen und Blutun-gen der - 194

- Störungen der Zirkulation der - bei Polvzythämie 196

Retina, Oxydations-Reduktions-

Potential der - 177 Retinarisse, Verschluß der- 114,

191 Retinitis albuminurica 276

cireinata, pathologische Ana-tomie der -- 113

exsudativa interna 252

- pigmentosa, endokrine Befunde bei - 487

- $\quad$ stellata transitoria 197Ringstarlinse, angeborene fami- 
liäre - 192 Rinnenbildung, degenerative -

der Hornhäute 252 Röntgendiagnostik 208 (Bb.) Röntgenstrahlen, Wirkung der

- auf das Auge 173

- $\quad$ bei Iritis haemorrhagica 397

- $\quad$ Prothese zum Schutze gegen -487

Röntgenstrahlenkatarakt, ex-

perimenteller 289 Röntgentherapie 208 (Bb.)

der Granulose 441

der Keratitis 419

- $\quad$ extraokulärer Tuber kulose 190Rosacea, Verhältnis der- zum U1-

cus corneae rodens 203

$\mathrm{S}$

Sarkoid Boeck, symmetrisches - der Conjunctiva 334

Scharlachrotfärbung der Hornhaut und Bindehaut Ill

Schielen 84ff., 407, 467

- $\quad$ Sehschärfe beim schielenden Auge

374

- $\quad$ stereoskopische Bilder für schie-lende Kinder 199

Strahlenbehandlung der Tuber-

kulose des Auges 403 Schlaf krankheit, Keratitis paren-

chymatosa bei -- 175 Schlemmscher Kanal, Verbin-

dung des - nach außen mit hoch-

gradiger Hypotonie des Augapfels

487 Schule, augenärztliche Tätigkeit in

der - 398 Schwangerschaft, Einfluß der -

auf den intraokularen Druck 382,

386 Schwimmbadconjunctivitis332 Sehnerv s. Nervus opticus Sehschärfe beim schielenden

Auge

374

Abhängigkeit der - von der Be-leuchtungsstärke 288

der Automobilführer, Anforde-rungen für die -- - in der Schweiz $\tau$ เo

Sachregister zu Bd. 66.

499

Serodiagnose, Bedeutung der -in der Therapie des Auges 385

Serum, Elektrolytgehalt des - 138

Serumultrafiltrat, Elektrolytgehalt des -138

Siderosis bulbi 376

Siderosis corneae 345

Sinus sphenoidalis, Trepanation des - bei Neuritis optica 292

Skier a, pathologische Anatomie der

- $336 f f$.

- $\quad$ blaue - und Knochenbrüchigkeit

397. 456 Skleralnaht 395 Skleritis, Schlodtmannsche 356 Skotome, Nachweis kleiner - 378

Sondermannsche Trepanation

des Ulcus serpens 345 Sonnenblumenstar 106 Soziale Versicherung, Schäden 
der - und Wege zu ihrer Besse-

rung 419 (Bb.) Staehlische Linie, eigenartigeVer-

hältnisse in der Gegend der - 201 Staphylombildungen der Horn-

haut 455 Strabismus s. Schielen Sympathikus, Symptome bei Reiz

des zervikalen - 384 Sympathisierung des Kaninchen-

auges 364 Synchisis nivea 367 - scintillans, Schädigung durch

- 474

Syphilis des Auges 386, 418 (Bb.)

der Bindehaut 333

Mißerfolge der kombinierten Ar-sen- und Wismutbehandlung bei

- 413

Syphilis hereditaria, Hydrar-

throse bei - 385 Syphilome, multiple - des Auges

375

- $\quad$ des Auges in der dritten Generation 489

$\mathrm{T}$

Tarsektomie gegen Entropium

senile 396 Tarsitis syphilitica 410 Tätowierung der Hornhaut 344 Teerbepinselung, Reaktion der

Hornhaut auí - 344 Thoraxquetschung, sofort ein-

setzende Blindheit bei - 205 Thorium X, therapeutische Ver-

wendung des - 172 Tonus und Raumwahrnehmung 190 Tonusvalenz der chromatischen

Erregung bei der optomotorischen

Reaktion 135 Tophus syphiliticus des Tränen-

beins 404

Totische Operation 253, 257 Trachom 330

Beziehungen des - zur Tuber-kulose 386

mit Einschlußblennorrhoe geimpft 467

Röntgenbehandlung des - 441

Wirkung des Serums adenoider Trachomkranker 385

Behandlung des trach. Pannus mit dem Sekret der trach. Bindehaut 399

- $\quad$ und Unfallversicherung 100Tracumin 79

Tränenbein, Tophus syphiliticus

des - 404 Tränendrüse, Ektopie der - 269

- $\quad$ Mißbildung des Ausführungsgan-ges der - 454

- $\quad$ Mischgeschwülste der - 266Tränendrüsenentfernung,

schädliche Folgen der - 171

Tränenröhrchen, Konkrement-bildung im oberen - 485

Tränenwege, pathologische Anatomie der $-328 \mathrm{ft}$.

Trauma 369

Trepanation, Elliotsche, Beein-flussung der Topographie des Seh-nervenkopfes nach - 256

- $\quad$ oder Zyklodialyse 146Trichromaten, Einfluß psychologi-

scher Momente auf die farbigen Schwellen der anomalen - 133 Tuberkulose des Auges 273

der Bindehaut 332

der Iris 409

- der Sklera 347

- undsympathischeOphthalmie384 
- $\quad$ Beziehungen des Trachoms zur-386

- Beziehungen der Augen-T. zur tub. Allgemeinerkrankung 118, 119

- $\quad$ Strahlenbehandlung der - 403-- Röntgenbehandlung extraoku-

lärer - $190 \mathrm{Tu}$ berkulosevirus BCG, Cal-

mettesches 120 Typhus-Paratyphus-Vakzine,

intravenöse Anwendung der -

bei Augenkrankheiten 490

$\mathrm{U}$

Ulcus corneae rodens und sein Verhältnis zur Rosacea 203

Ulcus serpens und sympathische Ophthalmie 361

- $\quad$ Sondermannsche Trepanation des

“--- 345 ,, ,

Ultramikroskopie der lebenden

Hornhaut 259

$33^{*}$

500

Sachregister zu Bd. 66.

Unfallkunde 369, 415, 491 Uvea, pathologische Anatomie der $347 \mathrm{fi}$.

Vakzine, polyvalente - bei Augen-

infektionen 291 Vakzineinfektion des Auges 382 Velonoskiaskopie, Apparat für

die - 384 Verätzungen, Frühplastik mit

Mundschleimhaut bei - 263 Verfettung des Bulbus, Rolle der

Histiozyten bei der degenerativen

- 158, 163 Versicherung, Schäden der sozia-

len - und Wege zu ihrer Besse-

rung 419 (Bb.) Versicherungskunde 390, 415,

475. 49i Versicherungsmedizin 492 (Bb.) Vestibuläre Augenbewegungen,

Apparat zur Registrierung der

194 Vitaminfreie Nahrung, Kata-

raktbildung bei -365

Vitiligo 334

Vorderkammer, Verbindungder - nach außen mit hochgradiger Hypotonie des Augapfels 487

W

Wundsprengungen, Folgen der - nach Staroperation 396

Z

Zangengeburt, angeborene Horn-

hauttrübung nach $-484 \mathrm{Z}$ ell on, durchsichtiger Augenver-

band aus - 201 Ziliarepithel 349 Ziliargefäße, Veränderungen der

- bei Glaukom 196 Ziliarnervenschleifen, intraskle-

rale 59, 346, 459 Zirkulationslücke bei chronischer

Iridozyklitis 107 Zonulalamelle 365 Zyklodialyse, Modifikation der -

394 - oder Trepanation 146 Zyklopie 450

Namenregister zu Banð 66.

A 
Abadie 413 Abe 364 Aecardi 380 Agnello 369 Alajmo 378, 379, 386 Albrich 387 d'Amico 376, 378 Amsler 102 Archangelski 460 Angelucci384, 385,386 Argañasaz 467 Ascher 90, 145, 155 Aschoff 281 Ask 420 (P.) Asmus 90 Augstein 403, 404 Avizonis 171, 454 Axenfeld 346, 459 B

Bachstez 84 Bar 481 Barbato 384 Bartels 92, 293, 479, 480, 482 Baumert 203

Baurmann 138, 151

Beck 119

Becker 120, 255, 256

de Benedetti 377, 454

Berger 342,375

Best 126, 128

Birch-Hirschfeld275,

287, 290, 330, 404,

445 Bistis 334 v. Blaskovics 165, 391,.

394- 395. 396, 397.

399 Blatt 489 Blum 461 Boenheim 487 Bornstein 281 Borrello 375 Brons 479 Bruckner 273

Brunner 392 Busacca 344, 382 Busse 159, 340

C Cameron 458 Casolino 385

Caspar 462 Charschak 409 Clausen 132, 253, 254, $256,258,264,270$,

284 Colden 456 Comberg 116, 128, 132,

151, 20T, 282, 286 Cords 97, 114, 117, 148 v. Csapody 398

$\mathrm{D}$

D. Damskaja 409 Davenport 453 Deutschmann 169,286 Dieter 300 Dimitriades 91 Dinger 261 484 v. Ditrói 393 Dobrinow 409 ten Doesschate 268 Dohlmann 194 Dombrowskaja 410

Dreyfus 205 Dubois 102 Dusseldorp 414 